\title{
Energy Efficient Buildings with Functional Steel Cladding
}

\author{
M. A. Joudi, M. Rönnelid, H. Svedung and E. Wäckelgård \\ School of Technology and Business Studies, SERC, Dalarna University, SE-791 88 Falun, Sweden \\ *Corresponding author.Tel: +46 23778738,E-mail: ewc@du.se
}

\begin{abstract}
The aim of the study is to develop a model for the energy balance of buildings that includes the effect from the radiation properties of interior and exterior surfaces of the building envelope. As a first step we have used ice arenas as case study objects to investigate the importance of interior low emissivity surfaces. Measurements have been done in two ice arenas in the north part of Sweden, one with lower and one with higher ceiling emissivity. The results show that the low emissivity ceiling gives a much lower radiation temperature interacting with the ice under similar conditions. The dynamic modelling of the roof in ice arenas shows a similar dependence of the roof-to-ice heat flux and the ceiling emissivity.

A second part of the study focus on how to realise paints with very low thermal emissivity to be used on interior building surfaces.
\end{abstract}

Keywords: Energy balance, Low emissivity, Radiation properties

\section{Introduction}

The need for buildings to be highly energy efficient requires that a building envelope must be air tight and well insulated. It is also important that both interior and exterior surfaces has appropriate optical functions. The exterior surfaces (roof and facades) should in a hot climate have high solar reflectance and high infrared emissivity in order to reduce space-cooling loads. Buildings in colder climates should have exterior surfaces with high solar absorptance and low thermal emissivity to reduce space-heating loads. Buildings in all types of climates should have interior walls and ceilings with low thermal emissivity which will reduce the radiation exchange between persons and interior surfaces and thereby the need for decrease/increase the air temperature in order to compensate for too high/low interior radiation temperature.

The research has been extensive in finding solutions for hot climates to prevent over heating and one concept here is the so called cool roof, works from Synnefa et al [1] and Levinson et al [2] shows that it is a large potential for reducing energy use for cooling by use of near infrared high reflectance exterior paints. There has not been as much focus on interior surfaces and how functional optical properties can influence the energy demand in hot or cold climates. Development in paint formulation has made it possible to produce coating with higher reflectance in the long wave radiation spectra. This kind of coatings has been found mainly useful for interior coatings where it is expected to act like a thermal barrier for the radiation energy potentially emitted or absorbed by the surface when the radiation temperature differs significantly from the panel surface temperature. For instance, Daoud et al [3] has shown that low emissive interior coatings can contribute to the energy savings in an in doors ice rink.

In a previous work by Joudi et al [4] introduces a model for calculating the effect of both interior and exterior optical properties of a horizontal roof sandwich panel in terms of net energy flux per unit area. Sandwich panels usually consist of two coil coated steel sheet profiles, which are tightly pressed and glued to an insulation core. They can be manufactured even without trans-sectional supports to avoid thermal bridges. The results from the study indicate potential energy saving by the smart choice of optical properties of interior and exterior surfaces. In the present report we introduce a model that includes a roof panel and a 
floor and vertical heat exchange. The model is applied on indoor ice rinks as a case study where it has been performed measurements.

Due to the importance of having low thermal emissivity of the interior surfaces a part of the study was devoted to investigate the potential to further reduce the thermal emissivity in low emitting paints for interior use applied on coil coated stainless steel in sandwich panes. The main components of the paint counted from the steel surface are the primer, and the paint layer comprising the binder, pigment and aluminium flakes. The aluminium flakes are used for enhancing the infrared reflectance and by that reduce the thermal emissivity.

\section{Methodology}

As a first step we have used two ice rinks situated in the north of Sweden (Luleå) as case study objects to investigate the importance of the emissivity of the ceiling for the ice conditions. One of the ice-rinks has a highly emissive coil-coated steel sheet interior roof whereas the other ice-rink has a low emissive galvanized steel sheet interior roof. These buildings are of comparable design with similar roof heat transfer values. In order to study the dependence of the radiation heat flux on the interior surface thermal emissivity in building interior spaces with considerable surface temperature differences, continuous measurements of surface-, air-, and radiation temperatures in the two different indoor ice-rinks were made. Surface- and air temperatures were measured using T-type thermocouples and radiation temperatures were measured using pyrometers facing the ceiling and the ice floor respectively. The measurement data were collected using Intab loggers.

To investigate the flux of thermal radiation from the ceiling to the ice in indoor ice rinks with both low and high emissive interior roofs surfaces, the simulation environments IDA SE and IDA ICE 4.0 are used to solve a system of non-linear equations simultaneously, based on the work presented in [4]. In this model both interior and exterior surface temperatures, as well as discrete cell temperatures through the roof panel are simultaneously solved and coupled in an hourly dynamic simulation with varying outdoor conditions (i.e. solar irradiance, ambient and sky temperature) and different inside air and interior radiation temperatures (i.e. a radiation temperature as seen by the interior roof surface). Table 1 shows the model input parameters. Note that the location in the simulation is Stockholm, due to presently lack of data for Luleå.

Table 1. Model input parameters

\begin{tabular}{llll}
\hline Location & \multicolumn{3}{l}{ Stockholm } \\
\hline \multirow{3}{*}{ Insulation } & thickness & 0.3 & $\mathrm{~m}$ \\
\cline { 2 - 4 } & Thermal conductivity & 0.036 & $\mathrm{Wm}^{-1} \mathrm{~K}^{-1}$ \\
\cline { 2 - 4 } & Density & 20 & $\mathrm{Kgm}^{-3}$ \\
\cline { 2 - 4 } & Specific heat & 750 & $\mathrm{Jkg}^{-1} \mathrm{~K}^{-1}$ \\
\hline \multirow{2}{*}{$\begin{array}{l}\text { Exterior roof } \\
\text { optical properties }\end{array}$} & Total Solar Reflectance & 0.3 & - \\
\cline { 2 - 4 } & Thermal Emittance & 0.9 & - \\
\hline Ground reflectance & & 0.7 & - \\
\hline Air temperature under the ceiling & 12 & ${ }^{\circ} \mathrm{C}$ \\
\hline \multicolumn{2}{l}{ Ice surface Temperature } & -4 & ${ }^{\circ} \mathrm{C}$ \\
\hline
\end{tabular}

Effective emittance, $\varepsilon_{\text {eff }}$ with embodied view factor, $f_{c i}$ are calculated from Eq. (1) where $\varepsilon$ and $A$ are long wavelength emittance and area in $\mathrm{m}^{2}$ and subscript $c$ and $i$ represent ceiling and ice, respectively [5] 
$\varepsilon_{\text {eff }}=\left[\frac{1}{f_{C i}}+\left(\frac{1}{\varepsilon_{C}}-1\right)+\frac{A_{C}}{A i}\left(\frac{1}{\varepsilon_{i}}-1\right)\right]^{-1}$

The emittance of the low emissivity interior paint and its main components were determined from optical measurements of reflectance in the infrared wavelength range. For the primer, binder and the full paint formulation a Bruker Tensor 27 FTIR with gold-coated integrated sphere was used to measure in the wavelength range 2.5 to $20 \mu \mathrm{m}$. The flakes could be measured individually using a Hyperion microscope attached to the Technor FTIR. The thermal emissivity is calculated as an average emittance weighted by the black body radiation distribution for room temperature.

The binder was applied with a metal roll stick on optically smooth aluminium substrates. The thickness of the coatings was varied using different applicator rods. The primer was also applied on the same type of aluminium substrate and roll technique. The whole paint formulation was applied on stainless steel in a full-scale roll coating industrial process.

\section{Results}

Surface- and radiation temperatures measured in the two ice-rinks during a couple of warm summer days clearly indicate a reduced radiation heat flux from the ceiling to the ice floor in the low emissivity interior roof ice-rink compared to that in the ice-rink with the highly emissive interior roof surface. In Fig. 1a, it can be seen that the radiation temperature seen by the upwards looking pyrometer in the highly emissive interior roof surface ice-rink is very close to the interior roof surface temperature measured by a thermocouple mounted in contact with the surface. In contrast, as shown in Fig. 1b, in the ice-rink with the low emissive interior roof surface the corresponding radiation temperature is much lower and closer to the icetemperature.

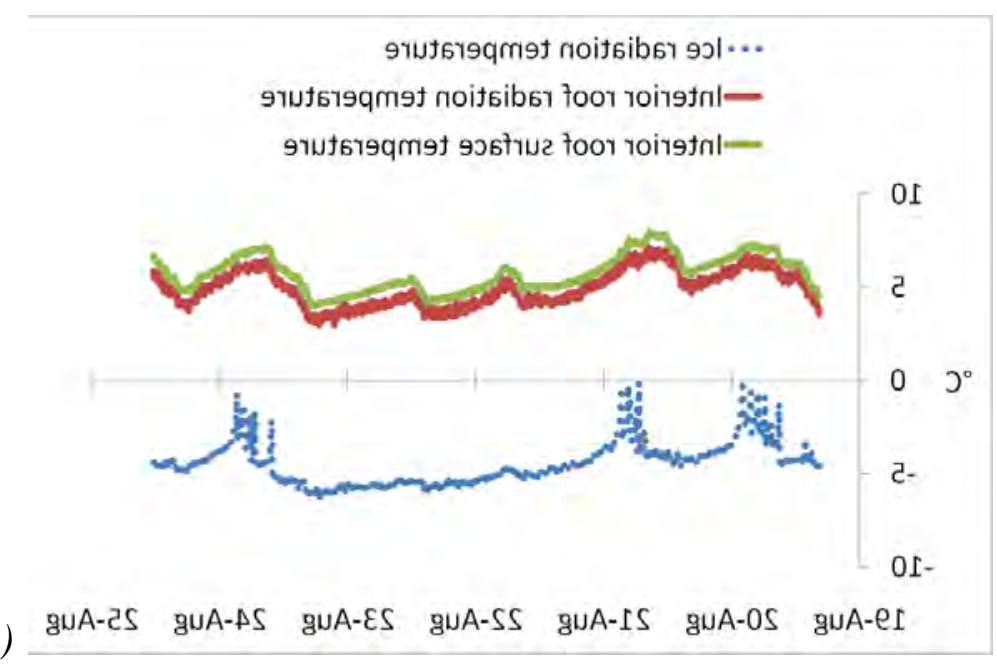

Fig. 1. The interior roof surface temperature, the radiation temperature measured by the upwards viewing pyrometer facing the interior roof surface and the radiation temperature measured by the downwards viewing pyrometer facing the ice are given vs. time during a series of summer days in a) the highly emissive interior roof surface ice-rink. 


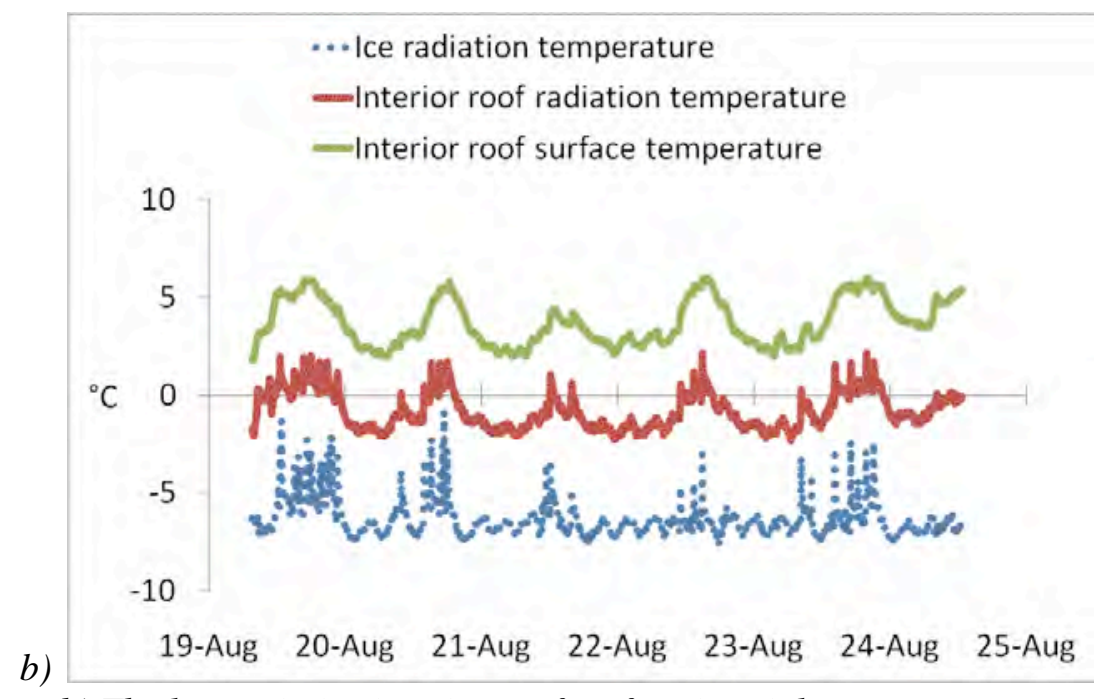

b) The low emissive interior roof surface ice-rink.

Calculations with the dynamic model show in Fig. 2 the thermal radiation to the ice surface, on monthly basis, for three different sets of effective emittance values of the ceiling. It shows that using low emissive coating on the roof interior decreases the radiative heat dissipation to the ice surface, resulting in less cooling load to maintain the ice surface at desired temperature. On the other hand, the monthly variation in the low emissive ceiling are almost negligible, arguably as the interior air temperature is maintained constant.

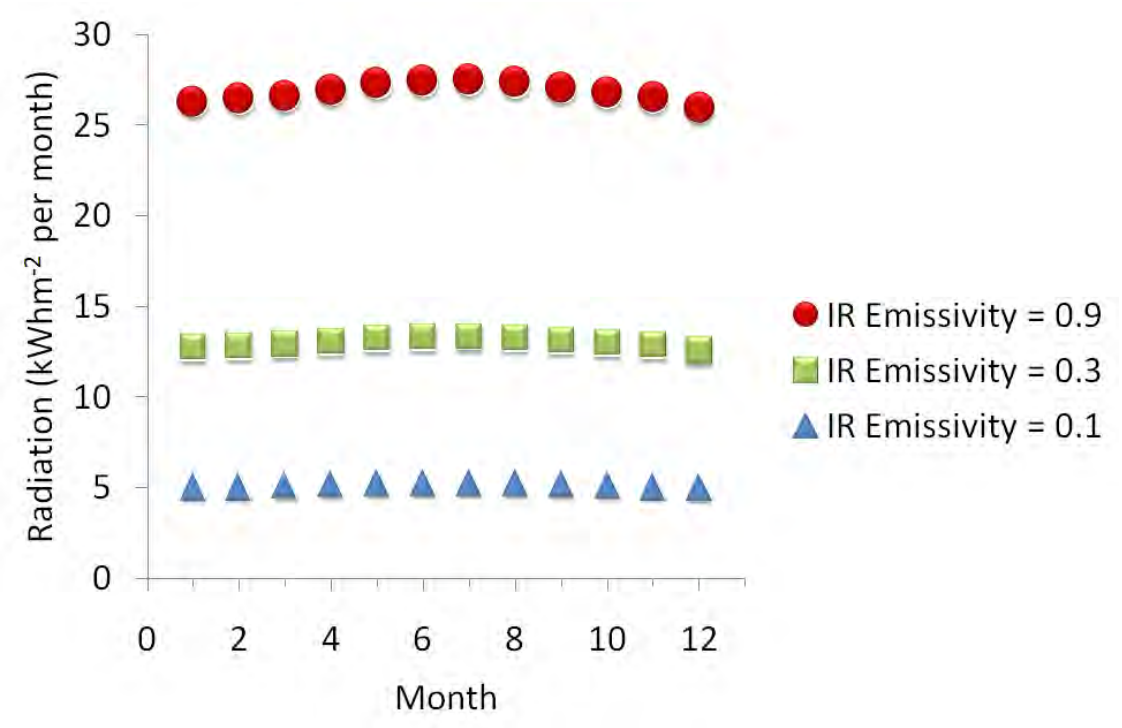

Fig. 2. Thermal radiation to the ice surface for each month for a ceiling for Stockholm climate.

Combining a high TSR (total solar reflectance) exterior roof coating and low emissive interior can reduce the total heat flux into the building; increasing the total solar reflection, TSR of the exterior coating reduces the solar gain, thus total heat flux into the building (heat surplus) will decrease. Further more, low emissive interior, reduces the radiation heat dissipation to the interior surfaces as demonstrated in the Fig 3. 


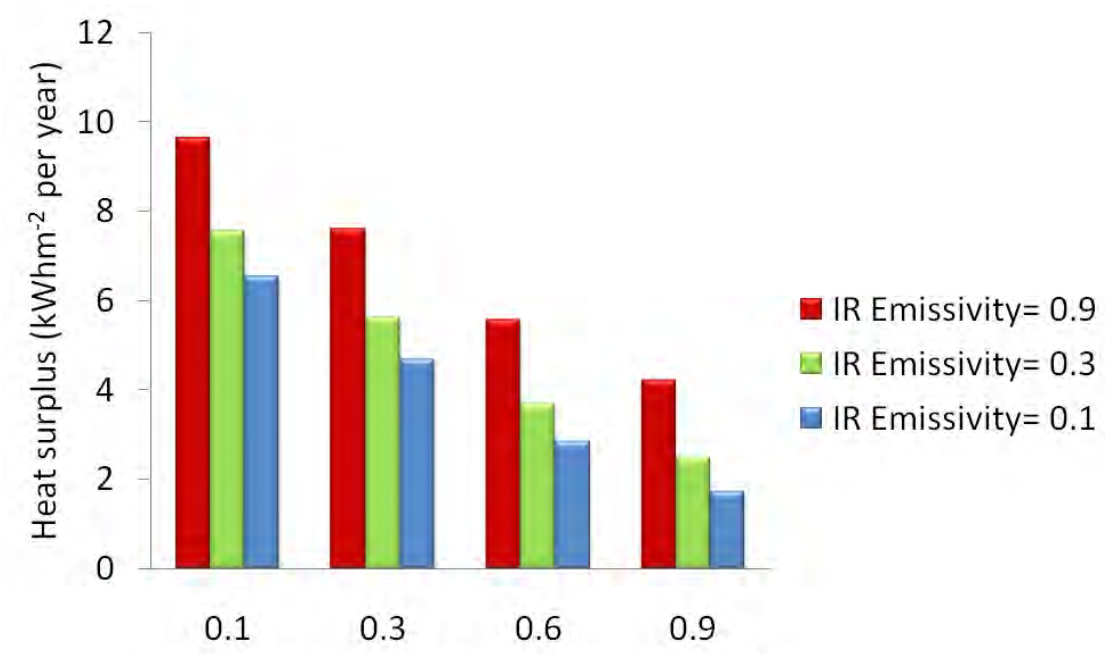

Exterior Roof Total Solar Reflectance, TSR

Fig. 3. The effect of combined different infrared emittance interior and high TSR exterior coatings on the heat surplus (cooling load) per roof area for Stockholm climate.

The reflectance measurements of the paint components are shown Fig. 4. The reflectance of the flakes cannot be measured for the longest recorded wavelengths due to the limited flake size (about $50 \mu \mathrm{m}$ on average). It is notable that the flake reflectance is about 0.90 (emittance 0.10) as the best case for the shorter wavelength, which is considerable lower than for a smooth aluminium surface (0.05). It is seen by visible inspection in the FTIR-microscope that the flakes have surface defects that will cause a reduced reflectance. The binder, which is a 3micrometer thick polyester shows a thermal emittance of 0.4 . Results from optimization of the paint system shows an emittance of about 0.35 for an optimized paint layer. The optimised paint contains $25 \%$ volume fraction of flakes and is about $7 \mu \mathrm{m}$ thick. The quality of the aluminium flakes that are used in the paints as emissivity-reducing components has also been studied. It was found that the paint with non-leafing type of flakes had lower emissivity than paints with leafing type.

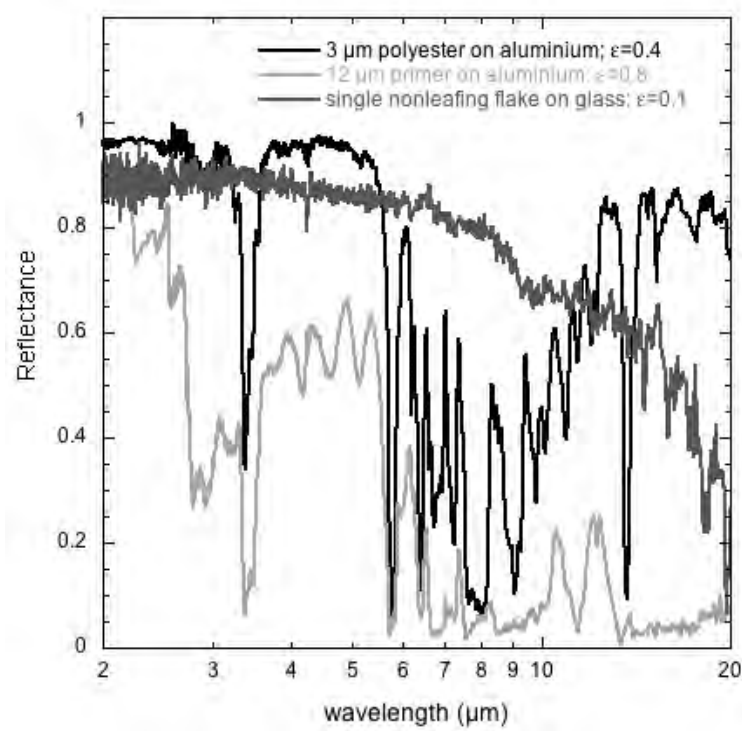

a)

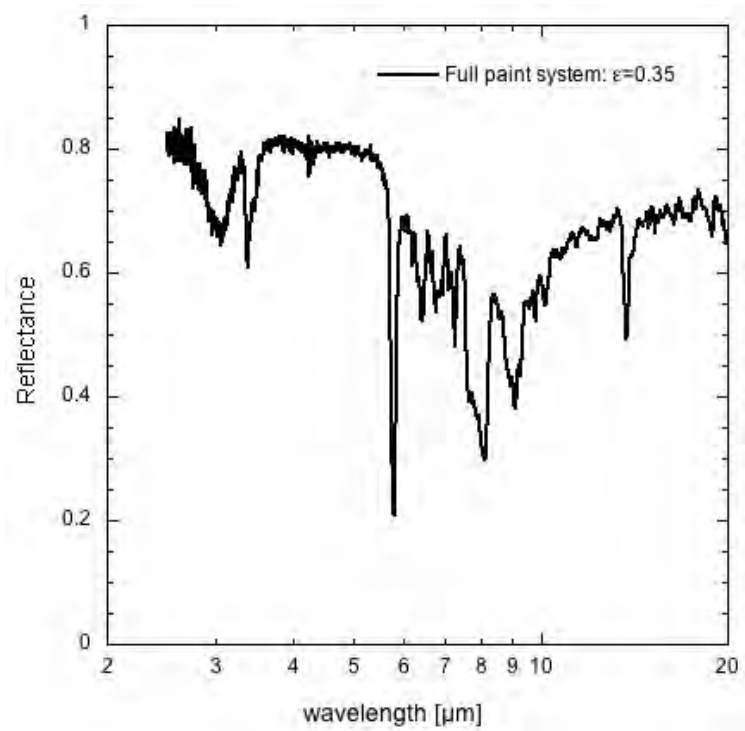

b)

Fig. 4. The reflectance measured on a) the main paint components: a single non-leafing aluminium flake, polyester binder on optical smooth aluminium and primer on the same substrate and b) a complete paint with the same types of components on galvanised steel. 


\section{Discussion and Conclusions}

The results show the importance of using low-emitting interior surfaces in such applications as indoor ice-rinks. The ice-rink measurements have so far only been presented for a few summer days but are now continuously being measured in order to cover a whole year. The dynamic model of the roof for vertical heat transfer gives an estimate of the amount of energy involved in the radiant transfer from ceiling to ice and also the amount of saved energy on an annual basis. The model will be further developed in order to account for dynamic effects from the convection in the interior space and how it relates to the emissivity of the ceiling. There is obviously a potential to save energy in ice-rinks using low-emissivity paints instead of ordinary paints and it is therefore important to continue the work on paints to reach as low emissivity as possible. However the low-emissivity paints developed so far has about twice the emissivity of a pristine galvanised steel surface and work is needed to develop binders with weaker infrared absorption (especially in the range 8 to $10 \mu \mathrm{m}$ ) and flakes with higher reflectance.

\section{References}

[1] A. Synnefa, M. Santamouris, and H. Akbari, Estimating the effect of using cool coatings on energy loads and thermal comfort in residential buildings in various climatic conditions, Energy and Buildings 39, 2007, pp. 1167 - 1174

[2] L. Levinson, H. Akbari, and J. C. Reilly, Cooler tile-roofed buildings with near-infraredreflective non-white coatings, Energy and Buildings 42, 2007, pp. 2591- 2605

[3] A. Daoud, N. Galanis, and O. Bellache, Calculation of refrigeration loads by convection, radiation and condensation in ice rinks using a transient 3D zonal model, Applied Thermal Engineering 28, 2008, pp. 1782-1790

[4] A. Joudi, H. Svedung, M. Rönnelid, Energy Efficient Surfaces on Building Sandwich Panels - A Dynamic Simulation Model, submitted to Building and Energy September 2010

[5] ASHRAE Handbook of Refrigeration - SI Edition 2006

\section{Acknowledgement}

The study has been funded by The Knowledge foundation in Sweden (KK-stiftelsen) and in cooperation with SSAB and Plannja AB. The authors would also like to thank Irina Tran at Akzo Nobel and Thomas Forsberg Luleå Technical University for contributions in the project. 